\title{
Reflections on epistemological aspects of artificial intelligence during the COVID-19 pandemic
}

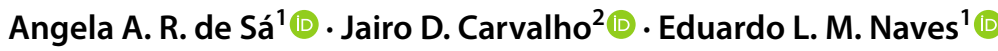

Received: 23 March 2021 / Accepted: 5 November 2021

(c) The Author(s), under exclusive licence to Springer-Verlag London Ltd., part of Springer Nature 2021

\begin{abstract}
Artificial intelligence plays an important role and has been used by several countries as a health strategy in an attempt to understand, control and find a cure for the disease caused by Coronavirus. These intelligent systems can assist in accelerating the process of developing antivirals for Coronavirus and in predicting new variants of this virus. For this reason, much research on COVID-19 has been developed with the aim of contributing to new discoveries about the Coronavirus. However, there are some epistemological aspects about the use of AI in this pandemic period of Covid-19 that deserve to be discussed and need reflections. In this scenario, this article presents a reflection on the two epistemological aspects faced by the COVID19 pandemic: (1) The epistemological aspect resulting from the use of patient data to fill the knowledge base of intelligent systems; (2) the epistemological problem arising from the dependence of health professionals on the results/diagnoses issued by intelligent systems. In addition, we present some epistemological challenges to be implemented in a pandemic period.
\end{abstract}

Keywords Espistemology $\cdot$ COVID-19 $\cdot$ Artificial intelligence $\cdot$ Intelligent systems

\section{Introduction}

We already know that Coronavirus SARS-CoV-2 has infected thousands of people, caused deaths and continues to spread throughout the world (PHAM et al. 2020). In this context, a specific digital technology called Artificial Intelligence (AI) plays an important role and has been used by several countries as a health strategy in an attempt to understand, control and find a cure for the Coronavirus disease (COVID-19) caused by this virus (DSouza et al. 2019; Kulkarni et al. 2020).

Many efforts and investments have been applied to the AI research area to combat the COVID-19 pandemic. Intelligent

Angela A. R. de Sá

angelaabreu@gmail.com

Jairo D. Carvalho

jairodc_8@hotmail.com

Eduardo L. M. Naves

eduardonaves@ufu.br

1 Assistive Technology Group, Faculty of Electrical Engineering, Federal University of Uberlândia, Uberlândia, Brazil

2 Technologies Study Group, Faculty of Philosophy, Federal University of Uberlândia, Uberlândia, Brazil computing systems can support health decision-making when problems are complex, when there is still not much information about a new virus and when the time requires rapid results, as is the context of a pandemic (Adly et al. 2020). In this sense, AI systems can assist in accelerating the process of developing antivirals for Coronavirus and in predicting new variants of this virus (Adly et al. 2020; Ahuja et al. 2020). For this reason, much research on COVID-19 has been developed with the aim of contributing to new discoveries about the Coronavirus (Albahri et al. 2020; Lalmuanawma et al. 2020; PHAM et al. 2020).

However, there are some epistemological aspects about the use of AI in this pandemic period of COVID-19 that deserve to be discussed and need reflection. An important epistemological aspect concerns the use of real data that is needed to fill the knowledge base of AI systems for the discovery of new Coronavirus information. Patient data and clinical information must be used for an AI algorithm to produce machine intelligence ( $\mathrm{Li}$ et al. 2018; Al-Rubaie 2019). In addition, biomedical data can be obtained through sensors connected to a patient's body (electromyographic, electroencephalographic, galvanic skin response, temperature, respiratory rate, etc.) and be inserted into an AI system (Swapnarekha et al. 2020). Would it be reasonable, when it comes to data privacy, to use patient data to get quick results 
from an intelligent system in a pandemic period? In addition, we have another important epistemological aspect in relation to the dependence of medical professionals on the results/ diagnoses provided by intelligent systems. What would be the boundary between artificial knowledge of intelligent systems and scientific knowledge of health professionals? Is it worrying that we need to have rapid results in a pandemic period and, therefore, the medical field to become hostage to diagnoses issued by intelligent systems? Thus, it is important to study these epistemological aspects of the application of $\mathrm{AI}$ in the development of new health technologies and their impacts on the lives of health professionals and patients during the COVID-19 pandemic period.

In this scenario, this essay presents a reflection on the two epistemological aspects faced by the COVID-19 pandemic: (1) The epistemological aspect resulting from the use of patient data to fill the knowledge base of intelligent systems; (2) the epistemological problem arising from the dependence of health professionals on the results/diagnoses issued by intelligent systems. To discuss these epistemological aspects of the application of AI in the context of Coronavirus, we will first present an overview of the use of intelligent systems that are helping to combat COVID-19. Next, we will explain about the epistemological aspects of $\mathrm{AI}$ in the context of the Coronavirus pandemic and the possible impacts on our lives. In conclusion, we will present some challenges of using $\mathrm{AI}$ in pandemic periods.

\section{Materials and methods}

To carry out the search for articles for the scientific basis of the epistemological reflection proposed in this article, we used the criteria for Preferred Reporting Items for Systematic Reviews and Meta-Analyses (PRISMA) (Moher et al. 2009a, b). The entire study selection process was carried out by three reviewers and summarized in a PRISMA flow diagram (Fig. 1).

\subsection{Inclusion/exclusion criteria}

Eligible articles accounted for studies on COVID using AI techniques. We excluded studies that did not present results of technological development to be used by medical professionals or did not present epistemological aspects of AI. The search included English and Portuguese language. Data collection took place up to May 2021.

\subsection{Search sources}

We performed the search of articles in three databases: Web of Sicence, Pubmed, and Scopus). For our study, the following search terms was used: [("Artificial intelligence") AND ("2019-nCoV" OR 2019nCoV OR nCoV2019 OR "nCoV2019” OR “COVID-19” OR COVID19 OR "HCoV-19" OR HCoV19 OR CoV OR "2019 novel*" OR Ncov OR "n-cov" OR "SARS-CoV-2" OR "SARSCoV-2" OR "SARSCoV2"
Fig. 1 PRISMA method used for the selection of articles for this study
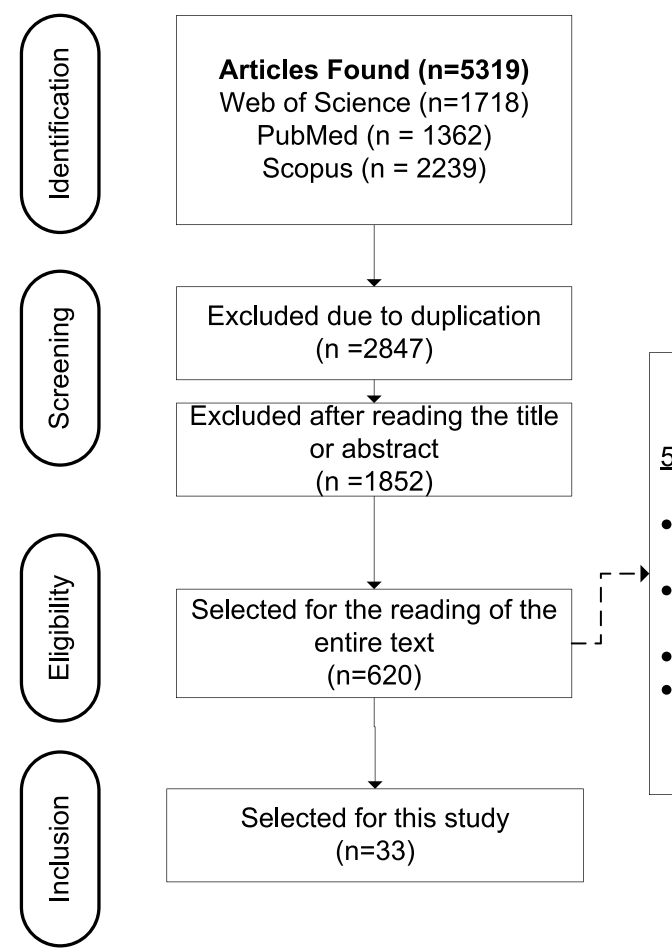

$\underline{587 \text { full-text excluded, with the reasons: }}$

- Did not present results of technological development to be used by health area; Did not present epistemological aspects of $\mathrm{Al}$;

- The full text was not available;

- Not written in English or Portuguese 
OR "SARSCoV2" OR SARSCov19 OR "SARS-Cov19" OR "SARS-Cov-19")]. We have chosen a wide query to have the chance of carefully screening the content and the subject of each article.

\subsection{Selection}

Two authors (AARS and JDC) independently analyzed the titles and abstracts of the studies identified by the research strategy and confirmed the existing duplicates to remove them. Eligible studies were selected after reading the full text to compose this study. Figure 1 depicts the PRISMA flow diagram selection process.

Two authors (AARS and JDC) extracted data from the selected studies and other (ELMN) verified and ratified the information. The search in the databases resulted in a total of 5319 articles. Duplicated were disregarded and 620 articles remained (Fig. 1). After reading the pre-selected studies, 587 were excluded after reading the full text due to the following reasons: (1) Did not present results of technological development of AI to be used by health area; (2) did not present epistemological aspects of AI; (3) The full text was not available; (4) not written in English or Portuguese. Therefore, the sample for this study consisted of 33 studies (Fig. 1).

In addition, we considered the Population, Intervention, Control, Outcome and Study Design (PICO) (Brunnhuber et al. 2006; Moher et al. 2009a, b) approach to design the study selection:

1. Population: AI-developed technologies;

2. Intervention: Potential application in the health area in the context of COVID-19;

3. Control: None taken into account;

4. Outcome: Development of technologies using AI techniques;

5. Study design: Methodology with a focus for support in the context of COVID-19.

\subsection{Results}

The 33 articles selected for our study are related to three application areas of AI in healthcare area that could or are applied in the context of COVID-19: algorithm techniques, robot development and epistemological approach, as depicted in Table 1. The next sessions will present a reflection on epistemological aspects of AI during the COVID-19 pandemic, using this selection of articles as scientific basis.

\section{Artificial intelligence versus COVID-19}

Artificial intelligence systems, which are composed of scientific techniques used for the implementation of Expert Systems and Knowledge Based Systems are increasingly used and are very important in the health area. (DSouza et al. 2019). The area of Machine Learning and Deep Learning, which is a specificity within Artificial Intelligence, are being highlighted in the evolution of technology in health, especially in this period of pandemic because it is being used to assist in the prediction and diagnosis of COVID-19 (Lalmuanawma et al., 2020).

In recent months, several AI applications have been developed and used to try to help combat Coronavirus: prediction, prevention, treatment, detection, diagnosis, mortality projection, vaccine development, identification and tracking (Hussain et al. 2020; Kannan et al. 2020; PHAM et al. 2020; Yu et al. 2020). According to (PHAM et al. 2020), the most important solutions of IA to combat the COVID-19 pandemic are early treatment and prediction.

Moreover, in relation to the antiviral development process for COVID-19, AI algorithms could be trained using data from the CORD-19 dataset and the results could then be used to track possible drugs that demonstrate efficacy in the treatment of COVID-19 (Ahuja et al. 2020). In addition, many AI algorithms extract information from scientific journals and help healthcare professionals estimate probabilities
Table 1 Selected articles as a scientific basis for the reflection on the epistemological aspects of AI proposed in our study

\begin{tabular}{ll}
\hline Application of AI in healthcare & Selected studies \\
\hline Algorithm techniques & Alanazi et al. (2017); Li et al. (2018); Al-Rubaie (2019); DSouza \\
& et al. (2019); Ahuja et al. (2020); Lin and Hou (2020); PHAM \\
& et al. (2020); Rasheed et al. (2020); Swapnarekha et al. (2020); \\
& Vaishya et al. (2020); Yu et al. (2020); Albahri et al. (2020); \\
& Bjerring and Busch (2020); Hussain et al. (2020); Kannan et al. \\
& (2020); Kulkarni et al. (2020); Lalmuanawma et al. (2020) \\
& Kulkarni et al. (2020), Tolsgaard et al. (2020), Vaishya et al. (2020) \\
& Boden (1978); Rowell (1989); Prem (2010); Russo (2018); Vascon- \\
& celos etal. (2018); Li et al. (2018); Al-Rubaie (2019); Schubbach \\
& (2019); Chin-Yee and Upshur (2019); Floridi (2019); Landgrebe \\
Robot development & and Smith (2019); PHAM et al. (2020); Schmetkamp (2020); \\
Epistemological approach & Walmsley (2020); Hagendorff and Wezel (2020); Hauer (2020); \\
& Luciano (2020); Niet and Bleakley (2020)
\end{tabular}


of specific diseases and suggest possible variations of the virus (Bjerring and Busch 2020).

In addition, AI robots have been used successfully in hospitals. Intelligent robots are being used to reduce the risk of healthcare workers' exposure to the virus: they are being deployed to handle and dispose of waste in hospitals (Kulkarni et al. 2020). Also, many robots are being used in the teleservice area to assist patients regarding the symptoms and procedures that they should be aware of in relation to COVID-19 (Vaishya et al. 2020).

Furthermore, AI systems have the ability to self-learn from new knowledge about the virus, which means that the intelligent system can improve itself when new data are added to its knowledge base (Lin and Hou 2020). And this characteristic of AI systems has been fundamental in this time of the COVID-19 pandemic, where all information is urgent, there is a great need for information and quick results so that they can help health professionals and government officials to choose the best one direction to protect the population against Coronavirus.

According to (Rasheed et al. 2020), AI systems have proved to be a highly versatile and powerful tool for managing coronavirus. However, while AI helps to track the data generated from the Coronavirus and that data are useful in predicting future outbreaks of this pandemic (Albahri et al. 2020; Kulkarni et al. 2020), there are philosophical and epistemological issues that need to be properly discussed.

\section{Epistemological aspects}

The Coronavirus-19 pandemic had a direct influence on the global economy and, undoubtedly, in the health area. The technology of intelligent systems is extremely important for scientific development in the medical field, assisting in the development of medicines, new diagnostic support systems and equipment for monitoring and treatment of newly discovered diseases/viruses. However, despite the urgency we have with the research results, it is important to reflect on the epistemological aspects of the overuse of intelligent systems in this pandemic period.

According to Piaget (Piaget 1970), "The problem of epistemology is to explain how real human thought is capable of producing scientific knowledge". And considering the context of intelligent systems, Schmetkamp (Schmetkamp 2020) argued that epistemological approach helps us to clarify whether we can empathize with AI.

\subsection{Epistemological aspect: healthcare professionals $\times \mathbf{A l}$}

We have to keep in mind that AI has the potential to improve the planning, treatment and notifications of patients with
COVID-19 (Vaishya et al. 2020). Several studies have shown that the use of AI can greatly improve the quality and accuracy of prediction, control and research in this area. (Lalmuanawma et al. 2020). However, due to the rapid increase in the amount of Coronavirus-infected patients in a short time, it becomes very difficult for the healthcare professional to complete the diagnostic process within an acceptable time (Swapnarekha et al. 2020). For this reason, AI plays an important role in the area of image processing as it assists medical staff in diagnosing images in a record time (Swapnarekha et al. 2020).

On the other hand, the inclusion of AI systems in medical decision-making may seem like an epistemic loss in medical understanding about issuing diagnoses and exam analysis. Considering that AI systems are important and effective in making medical decisions, could these intelligent machines epistemically outperform human medical professionals?

According to (Bjerring and Busch 2020), AI systems are black box that have reliability, precision and knowledge. In the health area, this black box promotes greater precision and reliability in medical decision-making. Also, (Laat 2020) argued that decision-making systems are increasingly present in our lives. However, Bjerring and Busch (2020) warned that the overuse and dependence on these intelligent systems can have a high cost for health professionals: loss of understanding and medical explanation (Bjerring and Busch 2020). In this sense, (Niet and Bleakley 2020) stated that the black box of intelligent systems needs to be opened because AI can put health professionals in difficult situations by providing wrong solutions/diagnoses or with which they disagree. In addition, Vasconcelos et at (2018) warned that the use of black-box AI algorithms in all decision-making systems may have unpredictable consequences on people's lives (Vasconcelos et al. 2018).

It is important to highlight that the ability to explain and justify clinical decisions is fundamental in clinical medicine. According to Chin-Yee and Upshur (2019), providing reasons for clinical decisions or interpretations of a patient's illness is the primary epistemic responsibility of clinicians (Chin-Yee and Upshur 2019). Nevertheless, intelligent systems can assist medical professionals (Tolsgaard et al. 2020): there are ChatBots, where users describe patients' symptoms and these systems inform possible diagnoses and even suggestions for treatments.

Furthermore, on the epistemic side of the issue, there is a call for transparency of the internal content of these intelligent systems that make predictions and make decisions (Walmsley 2020). But in the comparison between the human brain and the machine brain, we need to agree that the machine brain database was powered by a human brain, despite there are some differences between the mental abilities of humans and the brain computers. Even though the powerful developments in the field of AI, brain humans are 
superior to brain computers in some tasks, such as language creativity, intuition, visual recognition of complex contexts, etc. (Hagendorff and Wezel 2020). AI theory can reproduce only a small part of human computational skills (Boden 1978).

We must always remember that the black-box knowledge base of an AI system was provided by a human and that the decision rules were also designed by a human. Therefore, a wrong solution provided by an intelligent system may have been created by a human being, because the algorithms that design the artificial intelligence of a machine are also designed by a human being. Considering this epistemological information, one question is: would a wrong result/ diagnosis provided by an intelligent system be only machine responsibility? We know that the responses of an intelligent system will be exactly what a human programmer designed for the functioning of this AI system (Schubbach 2019). But the fact is that the human being in the face of the wrong result of a machine must have the necessary knowledge to recognize the error of an intelligent system.

Given this context, a pertinent question is: how will the use of AI systems in medical decision-making address good healthcare practices? Also, since AI systems have effective results in the medical field, will medical professionals have an epistemic obligation to trust and use AI systems in medical decision-making?

In summary, we should consider that, in times of pandemic, rapid results are essential and necessary. And AI systems are fast, useful and effective in this regard. But the main concern is the possible dependence of medical professionals on the results of an intelligent system. If for some reason the place does not have AI systems available for diagnosis, the health professional needs to be able to establish all the necessary reasoning to provide the diagnosis to the patient. And also know how to recognize when an AI system provides a result that may not reflect the true clinical status of the patient.

\subsection{Epistemological aspect: real data $\times$ Al}

Another epistemological aspect concerns the data that are used by the intelligent system to execute its algorithms and provide the results. For intelligent systems to work properly, they need their knowledge base to be populated with real patient information. According to (Swapnarekha et al. 2020), the following data types were used in research on prediction, classification and forecasting of COVID-19: (a) Clinical data: information on daily positive cases, total number of patients recovered per day and the mortality rates in a country; (b) Online data: data available from online sources; (c) Biomedical Data: medical images such as Computed Tomography or X-ray.
Unquestionably, patient data and clinical information must be used by an AI algorithm to produce machine intelligence (Li et al. 2018; Al-Rubaie 2019). In addition, biomedical data are obtained using sensors connected to the patient's body (electromyographic, electroencephalographic signals, galvanic skin response, temperature, respiratory rate, etc.) (Swapnarekha et al. 2020). According to (Hagendorff and Wezel 2020), personal data can help and is often essential, as software engineers need to populate the database of an AI system in order for it to be reliable and work properly.

When talking about patient data, it is valuable to think about privacy. According to (Russo 2018), privacy problems of personal data are generated by all digital technologies and it is not an exclusive concern with intelligent systems. This theme refers to changes in ontological and epistemological thinking and with ethical implications (Russo 2018). In contrast, the author (Luciano 2020) remember that the protection of personal data is not an insoluble problem, as it would be feasible for an intelligent system to use anonymous data, recorded only on the cell phone that would be used exclusively to send alerts in case of contact with people infected with Coronavirus.

In addition, privacy techniques for AI systems already exist. These techniques use cryptographic approaches to protect private data when testing or training an AI algorithm (Al-Rubaie 2019). One of the main ideas of AI privacy approaches is to learn a rule from a data set without revealing much about a single individual (Li et al. 2018). Unfortunately, not all AI systems have techniques to protect private data.

Nevertheless, privacy policies are another issue as they could help data owners specify what data are being shared, who would use the data and for what purposes (Al-Rubaie 2019). Even with the need for real data processing from AI systems in the Coronavirus pandemic, a privacy policy could give the patient a chance to choose whether or not to participate in the knowledge base of an intelligent system and decide which data they would like to use and share to contribute to improving research against a particular disease.

\section{Discussion}

Based on the selected articles depicted in Table 1, we presented a reflection on epistemological aspects of $\mathrm{AI}$ that require attention during the COVID-19 Pandemic. However, the focus of this article is not to indicate new directions of AI, but to warn against overuse in relation to AI technologies (Bjerring and Busch 2020). This specific issue is high important not only for health professionals, but for the whole society. Thus, the scientific contribution of our paper is about reflecting on society's dependence and need on AI technologies. This is a significant epistemological aspect 
of science, to which society needs to start evaluating, discussing and understanding the best way to deal with AI not only at the present moment but in the very near future, in accordance with the remarks of (Makridakis 2017) about AI revolution in the coming years.

Faced with all the reflection we have presented in the previous sessions, we must be mindful that AI technologies are useful and greatly assist in the control and diagnosis during the Coronavirus pandemic, as demonstrated by (Hussain et al. 2020). According to the studies of (Awwalu et al. 2015; Hamet and Tremblay 2017) the use of AI techniques in medicine is important for accuracy in disease discovery, treatment and drug delivery. However, we need to emphasize that AI systems should be cognitive co-agents to assist human intelligence and not compete with it or even replace it (Jarrahi 2018). In line with the studies of (Horn 2001; Varlamov et al. 2019), AI systems are essential tools to assist the healthcare professional find a comprehensive picture of the patient's situation.

Moreover, the epistemological aspect of AI of assisting human intelligence also makes us reflect on how society is holding out all hope of replacing the work of a human being in AI, whether for a diagnosis, recognition etc. As we discussed in the previous section, $\mathrm{AI}$ is programmable by humans and the knowledge of an AI technology is also created by humans (Korteling et al. 2021). Therefore, diagnoses produced by AI, like those produced by humans, can also be erroneous or misleading in their results. In that context, it is essential to be careful with outcomes from AI technologies: they should assist us and not alienate us and prevent us from exercising human reasoning. In line with the statement of (Kundu 2021), healthcare professionals expect AI systems to help them enhance rather than replace their capabilities.

Furthermore, despite all the relevance for the health area and assistance in numerous applications to combat the pandemic, the use of patient data is also a worrying factor ( $\mathrm{Li}$ et al. 2018; Al-Rubaie 2019). Whether for research purposes or to feed the database of AI systems, the use of this data is necessary but we need to discuss more about transparency and authorization for the use of this data, whether personal, clinical or biomedical, as has pondered (Stahl and Wright 2018) on privacy and data protection in AI systems.

Nevertheless, what we actually have now are healthy questionings, concerns and discussions regarding the use and applications of AI systems. The benefit that these systems have already added to research and society is unquestionable. But we have to keep discussing and understanding the dynamics of society's relationship with AI systems. According to (Dignum 2017), AI systems will be taking decisions that affect our lives and our way of living in many different levels. The fact is that in the near future these systems will be increasingly embedded and integrated not only in the healthcare area, but in all sectors (Makridakis 2017).
Therefore, society needs to prepare itself to be able to get the best out of these AI systems, but not be totally dependent on them.

\section{Challenges and future}

Despite all the benefits that AI can bring to us in the Coronavirus pandemic, we need to keep in mind some of the inherent epistemological issues of these systems: accuracy of prediction and necessity of advance intelligent systems on symptom-based identification of COVID-19 (Kannan et al. 2020; Alanazi et al. 2017). In contrast, AI systems can have faults or erroneous results, which can lead to serious risks, depending on the technological context in which the applications are used (Hagendorff and Wezel 2020).

We have to recognize that AI has shown its potential in the battle against the COVID-19 pandemic. However, there are some epistemological challenges: there are no standard data sets for training the knowledge base and no privacy policy (Rowell 1989; Floridi 2019; Landgrebe and Smith 2019; PHAM et al. 2020; Walmsley 2020). Furthermore, there is no definition as to the excessive use of AI systems for diagnosis in the medical field.

Another epistemological challenge is the participation of a team of experienced experts to help define the behaviour of an intelligent system, thus avoiding error-prone data interpretation (Hauer 2020). However, despite the great potential of AI systems for diagnosis and treatment, they are not yet able to replace complex aspects of clinical care, such as intuition and clinical perceptions. Even so, the health professionals must learn to use AI systems without decreasing the ability to make their own evaluation of patients (Niet and Bleakley 2020).

Moreover, the epistemological reflection on the applications of AI that we presented in this article is also relevant to society in general. Considering that AI systems will increase more in the near future, society needs to start discussing harder about the benefits, impacts and the damages that the implementation of these systems may introduce in people's lives. Based on these discussions, it will be possible to design strategies to know how to deal and coexist in the best possible way with AI technologies.

Finally, researchers in general have to consider a new epistemological challenge: the practice of empathy, not only with intelligent robots, but also with humans (Prem 2010; Schmetkamp 2020). AI systems can conflict with the values and ideals of medical practice in various situations. We need to empathize to understand that sometimes there may be a reason for medical professionals to dispense with the use of AI systems and think critically, making their own decisions (Bjerring and Busch 2020). In this period of the Coronavirus pandemic, perhaps one of the biggest epistemological 
challenges really is to empathize with the choices and work of the professionals who are working exhaustively in the battle against this new virus.

Author contributions AARS, JDC and ELMN have been involved in drafting the manuscript and revising it critically for important intellectual content and given final approval of the version to be published. All authors read and approved the final manuscript.

Funding This study was financed in part by the Coordenação de Aperfeiçoamento de Pessoal de Nível Superior-Brazil (CAPES). Finance Code 001.

Data availability The dataset supporting the conclusions of this article is included within the article.

\section{Declarations}

Conflict of interest The authors declare that they have no conflict of interest.

Ethical approval Not applicable.

Consent to participate Not applicable.

\section{References}

Adly AS, Adly AS, Adly MS (2020) Approaches based on artificial intelligence and the internet of intelligent things to prevent the spread of COVID-19: scoping review. J Med Internet Res 22(8):115. https://doi.org/10.2196/19104

Ahuja AS, Reddy VP, Marques O (2020) Artificial intelligence and COVID-19: a multidisciplinary approach. Integr Med Res 9:1-3. https://doi.org/10.1016/j.imr.2020.100434

Alanazi HO, Abdullah AH, Qureshi KN (2017) A critical review for developing accurate and dynamic predictive models using machine learning methods in medicine and health care. J Med Syst. https://doi.org/10.1007/s10916-017-0715-6

Albahri AS et al (2020) Role of biological data mining and machine learning techniques in detecting and diagnosing the novel coronavirus (COVID-19): a systematic review. J Med Syst 44(122):1-11. https://doi.org/10.1007/s10916-020-01582-x

Al-Rubaie M (2019) Privacy-preserving machine learning: threats and solutions. IEEE Secur Priv 17(2):49-58. https://doi.org/10.1109/ MSEC.2018.2888775

Awwalu J et al (2015) Artificial intelligence in personalized medicine application of AI algorithms in solving personalized medicine problems. Int J Comput Theory Eng 7(6):439-443. https://doi. org/10.7763/IJCTE.2015.V7.999

Bjerring, JC, Busch J (2020) Artificial intelligence and patient-centered decision-making. Philos Technol. doi: https://doi.org/10.1007/ s13347-019-00391-6.

Boden MA (1978) Artifical intelligence and piagetian theory. Synthese 38:389-414

Brunnhuber $\mathrm{K}$ et al (2006) How to formulate research recommendations. BMJ 333(7572):804-806. https://doi.org/10.1136/bmj. 38987.492014 .94

Chin-Yee B, Upshur R (2019) Three problems with big data and artificial intelligence in medicine. Perspect Biol Med 62(2):237-256. https://doi.org/10.1353/pbm.2019.0012 de Laat PB (2020) Algorithmic decision-making based on machine learning from big data: can transparency restore accountability? Philos Technol. https://doi.org/10.1007/s13347-017-0293-z

Dignum V (2017) Responsible artificial intelligence: designing ai for human values. ITU J ICT Discov 1(25):1-9

DSouza DJ, Srivatsava S, Prithika R (2019) IoT based smart wheelchair for HealthCare. Int J Recent Technol Eng (IJRTE). https:// doi.org/10.35940/ijrte.B1592.078219

Floridi L (2019) What the near future of artificial intelligence could be. Philos Technol. https://doi.org/10.1007/s13347-019-00345-y

Hagendorff T, Wezel K (2020) 15 challenges for AI: or what AI (currently) can't do. AI Soc 35:355-365. https://doi.org/10.1007/ s00146-019-00886-y

Hamet P, Tremblay J (2017) Artificial intelligence in medicine. Metabolism. https://doi.org/10.1016/j.metabol.2017.01.011

Hauer T (2020) Machine ethics, allostery and philosophical antidualism: will ai ever make ethically autonomous decisions? Society. https://doi.org/10.1007/s12115-020-00506-2

Horn W (2001) AI in mediciente on its way from knowledge-intensive to data-intensive systems. Artif Intell Med 23:5-12. https:// doi.org/10.1016/s0933-3657(01)00072-0

Hussain AA et al (2020) AI techniques for COVID-19. IEEE Access. https://doi.org/10.1109/ACCESS.2020.3007939

Jarrahi MH (2018) Artificial intelligence and the future of work: human-AI symbiosis in organizational decision making. Kelley Sch Bus. https://doi.org/10.1016/j.bushor.2018.03.007

Kannan S et al (2020) The Role of artificial intelligence and machine learning techniques: race for COVID-19 vaccine. Arch Clin Infect Dis 15(2):1-9. https://doi.org/10.5812/archcid.103232

Korteling JE et al (2021) Human- versus artificial intelligence. Front Artif Intell 4:1-13. https://doi.org/10.3389/frai.2021.622364

Kulkarni P et al (2020) Utility of digital technology in tackling the COVID-19 pandemic: a current review. J Clin Diagn Res. https://doi.org/10.7860/JCDR/2020/45341.13919

Kundu S (2021) AI in medicine must be explainable. Nat Med. https://doi.org/10.1038/s41591-021-01461-z

Lalmuanawma S, Hussain J, Chhakchhuak L (2020) Applications of machine learning and artificial intelligence for Covid-19 (SARS-CoV-2) pandemic: a review. Chaos Solitons Fractals 139:1-6. https://doi.org/10.1016/j.chaos.2020.110059

Landgrebe J, Smith B (2019) Making AI meaningful again. Synthese. https://doi.org/10.1007/s11229-019-02192-y

Li P et al (2018) Privacy-preserving machine learning with multiple data providers. Future Gener Comput Syst. https://doi.org/10. 1016/j.future.2018.04.076

Lin L, Hou Z (2020) Combat COVID-19 with artificial intelligence and big data. J Travel Med. https://doi.org/10.1093/jtm/taaa080

Luciano F (2020) Mind the app—considerations on the ethical risks of COVID-19 apps title. Philos Technol. https://doi.org/10. 1007/s13347-020-00408-5

Makridakis S (2017) The forthcoming Artificial Intelligence (AI) revolution: its impact on society and firms. Futures 90(46):4660. https://doi.org/10.1016/j.futures.2017.03.006

Moher D et al (2009a) Preferred reportingitems for systematic reviews and meta-analyses: the PRISMA statement. BMJ 339(b2535):1-8. https://doi.org/10.1136/bmj.b2535

Moher D et al (2009b) Preferred reporting items for systematic reviews and meta-analyses: the PRISMA statement. PLoS Med (united States) 6(7):e1000097. https://doi.org/10.1371/journal. pmed.1000097

PHAM Q-V et al (2020) Artificial Intelligence (AI) and big data for coronavirus (COVID-19) pandemic: a survey on the state-ofthe-arts. IEEE Access 4:1-19. https://doi.org/10.1109/ACCESS. 2020

Piaget J (1970) Genetic epistemology. Columbia University Press, London 
Prem E (2010) Epistemological aspects of embodied artificial intelligence. Cybern Syst 28(5):3-9. https://doi.org/10.1080/01969 7297126047

Rasheed J et al (2020) A survey on artificial intelligence approaches in supporting frontline workers and decision makers for the COVID19 pandemic. Chaos Solitons Fractals 110(337):1-18. https://doi. org/10.1016/j.chaos.2020.110337

Rowell JA (1989) Piagetian epistemology: equilibration and the teaching of science. Synthese 80:141-160

Russo F (2018) Digital technologies, ethical questions, and the need of an informational framework. Philos Technol. https://doi.org/ 10.1007/s13347-018-0326-2

Schmetkamp S (2020) Understanding A.I.—can and should we empathize with robots? Rev Philos Psychol 11:881-897. https://doi.org/ 10.1007/s13164-020-00473-x

Schubbach A (2019) Judgingmachines: philosophical aspects of deep learning. Synthese. https://doi.org/10.1007/s11229-019-02167-z

Stahl BC, Wright D (2018) Ethics and privacy in AI and big data: implementing responsible research and innovation. Ethics Priv AI Big Data Implementing Responsib Res Innov 16(3):26-33. https://doi.org/10.1109/MSP.2018.2701164

Swapnarekha $\mathrm{H}$ et al (2020) Role of intelligent computing in COVID19 prognosis: a state-of-the-art review. Chaos Solitons Fractals 138:1-15. https://doi.org/10.1016/j.chaos.2020.109947

Tolsgaard MG et al (2020) The role of data science and machine learning in health professions education: practical applications, theoretical contributions, and epistemic beliefs. Adv Health Sci Educ 25:1057-1086. https://doi.org/10.1007/s10459-020-10009-8
Vaishya R et al (2020) Artificial Intelligence (AI) applications for COVID-19 pandemic. Diabetes Metab Syndr 14:337-339. https:// doi.org/10.1016/j.dsx.2020.04.012

van der Niet AG, Bleakley A (2020) Where medical education meets artificial intelligence: does technology care? Med Educ. https:// doi.org/10.1111/medu.14131

Varlamov OO et al (2019) Logical, Philosophical and ethical aspects of AI in medicine. Int J Mach Learn Comput 29(6):868-873. https:// doi.org/10.18178/ijmlc.2019.9.6.885

Vasconcelos M, Cardonha C, Goncalves B (2018) Modeling epistemological principles for bias mitigation in AI systems: an illustration in hiring decisions. In: AIES '18: proceedings of the 2018 AAAI/ACM conference on AI, ethics, and society. New York, NY, pp. 1-7

Walmsley J (2020) Artificial intelligence and the value of transparency. AI Soc. https://doi.org/10.1007/s00146-020-01066-Z

Yu P et al (2020) An application review of artificial intelligence in prevention and cure of COVID-19 pandemic. Comput Mater Cont 65(1):743-760. https://doi.org/10.32604/cmc.2020.011391

Publisher's Note Springer Nature remains neutral with regard to jurisdictional claims in published maps and institutional affiliations. 